\title{
Self-Assembly of CdTe Tetrapods into Network Monolayers at the Air/Water Interface
}

\author{
Matthew D. Goodman, ${ }^{\dagger}$ Lei Zhao, ${ }^{\dagger}$ Karen A. DeRocher, ${ }^{\dagger}$ Jun Wang, ${ }^{\dagger}$ Surya K. Mallapragada, ${ }^{\ddagger}$ and \\ Zhiqun $\operatorname{Lin}^{t, \pm, *}$ \\ ${ }^{\dagger}$ Department of Materials Science and Engineering and ${ }^{\ddagger}$ Department of Chemical and Biological Engineering, lowa State University, Ames, lowa 50011
}

S emiconducting nanocrystals, with their unique properties at the nanoscale, have received considerable attention due to their potential applications in solar cells, ${ }^{1-9}$ LEDs $^{10}$ tunable lasers, ${ }^{11}$ optical storage media, ${ }^{12}$ nonradiative energy transfer, ${ }^{13}$ biosensors, ${ }^{14,15}$ and bioimaging. ${ }^{16}$ Semiconductor nanocrystals with various shapes have been synthesized, with quantum dot $(\mathrm{QD})$, quantum rod ( $\mathrm{QR}$; i.e., nanorod), tetrapod, and nanowire synthesis being the most refined and controlled. While QDs are relatively easy to synthesize, properties that are more advantageous can be realized with the unique shapes. For example, the ability to manipulate the shape of nanocrystals has led to QRs with diameters ranging from 2 to $10 \mathrm{~nm}$ and lengths ranging from 5 to $100 \mathrm{~nm} \cdot{ }^{17,18}$ Because of their intrinsic structural anisotropy, QRs possess many unique properties that make them potentially better nanocrystals for photovoltaic cells and biomedical applications than QDs. They possess the polarized emission and their Stokes shift is dependent upon their aspect ratio. ${ }^{17,18}$ Photovoltaic cells made of QRs and conjugated polymers show an improved optical absorption in the red and near-infrared ranges originating from the QRs. ${ }^{3}$ Moreover, the long axis of the QRs provides continuous paths for transporting electrons. The performance of photovoltaic cells can be further improved if the QRs are vertically aligned between two electrodes to minimize the carrier transport pathways. ${ }^{3}$ The elongated nanorods and branched nanocrystals (i.e., tetrapods) enable more effective charge transport.

To date, there are few reports of nanocrystal assembly at the air/water interface. ${ }^{19,20}$ By contrast, studies have been www.acsnano.org

ABSTRACT Cadmium telluride (CdTe) tetrapods are synthesized with varying aspect ratios through multiple injections of the Te precursor, which provides an excellent means of controlling and tailoring the optical properties of the tetrapods. The self-assembly of CdTe tetrapods at the air/water interface is explored using the Langmuir-Blodgett (LB) technique due to potential use in solar cells arising from the intriguing tetrapod shape that improves charge transport and the optimum band gap energy of CdTe that enhances light absorption. Interestingly, the Langmuir isotherm shows two pressure plateau regions: one at $\sim 10 \mathrm{mN} / \mathrm{m}$ with the other at the high surface pressure of $\sim 39 \mathrm{mN} / \mathrm{m}$. LB deposition at various pressures allows the discernment of the unique two-dimensional packing alluded in the isotherm. By placing CdTe at the air/water interface, it is revealed in the deposition that the tetrapods experienced a dewetting phenomenon, forming a ribbon structure at the onset of surface pressure with a height corresponding to the length of one tetrapod arm. With the increase of surface pressure, the ribbons widen to an eventual large-scale percolated network pattern. The packing density of tetrapods is successfully manipulated by controlling the surface pressure, which may find promising applications in optoelectronic devices.

KEYWORDS: CdTe · tetrapod · self-assembly · network · Langmuir-Blodgett

conducted at the fluid/fluid interface, with one performed on nanorod assembly at the oil/water interface. ${ }^{21}$ By utilizing selfassembly at interfaces, for example, oil-water and air-water, two-dimensional organization of nanocrystals can occur, thereby providing a simple and effective route necessary for nanotechnology applications. Here we present a versatile method for cadmium telluride (CdTe) tetrapod synthesis by utilizing multiple Te precursor injections to provide the control over the tetrapod arm width, thereby effectively controlling the tetrapod shape. CdTe tetrapods are a promising inorganic semiconductor for photovoltaic cells due to its optimum band gap energy of $1.45 \mathrm{eV}$, high absorption coefficient of $10^{4} \mathrm{~cm}^{-1}$ for the visible solar spectrum, ${ }^{2}$ and additional three-dimensional nanostrutures for improved charge transport. As such, the CdTe tetrapods are placed at the air/water interface, experiencing a dewetting
*Address correspondence to
zqlin@iastate.edu.
Received for review February 7, 2010
and accepted March 24, 2010.
Published online April 1, 2010.
10.1021/nn1002584
@ 2010 American Chemical Society
043-2050 - 2010 


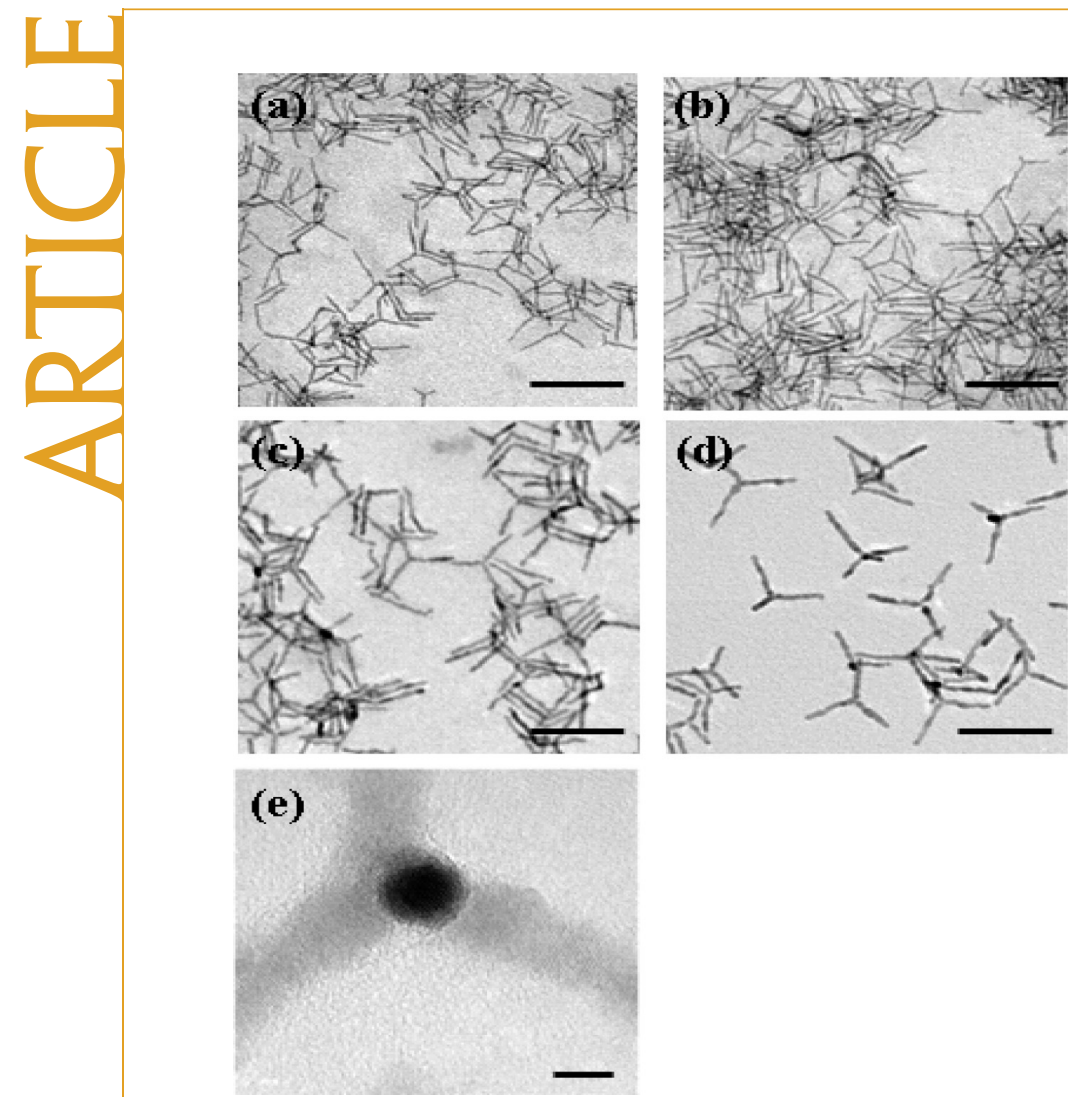

Figure 1. CdTe tetrapods synthesized via multiple injections of $\mathrm{Te}-\mathrm{TOP}$ in one reaction with aliquots taken after various number of injections: (a) 4, (b) 6, (c) 8, and (d) 10 injections. (e) Close up of a single tetrapod from panel d. Scale bars are $100 \mathrm{~nm}$ in panels a-d, and $5 \mathrm{~nm}$ in panel e.

phenomenon. This leads to the formation of a network monolayer structure at high pressures, which can be visualized optically. Insight into the unique synthesis of the CdTe tetrapods and the packing and rearrangement of the CdTe monolayer is gained. By using the facile and robust Langmuir-Blodgett (LB) deposition to systematically control the monolayer structure, the first

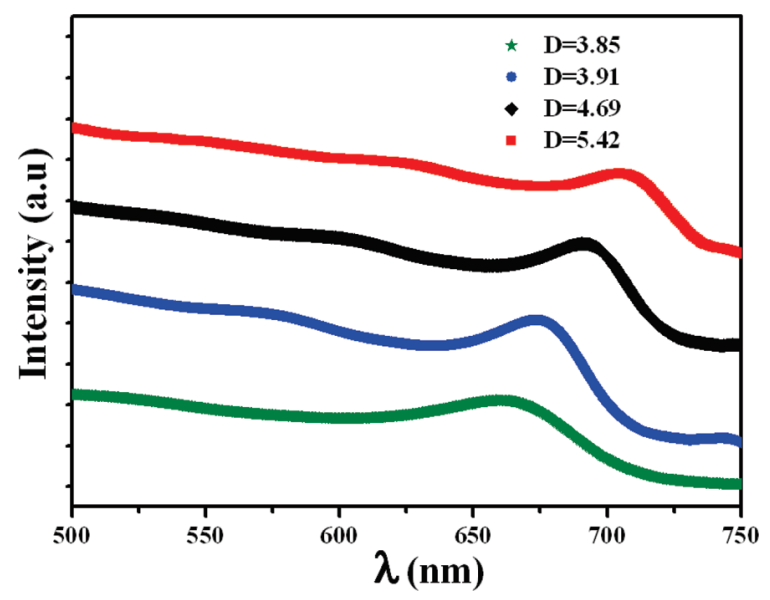

Figure 2. Absorption spectra of CdTe tetrapods made by multiple precursor injections: diameter, $D=3.85 \mathrm{~nm}(4 \mathrm{Te}-\mathrm{TOP}$ injections; green), $D=3.91 \mathrm{~nm}$ (6 Te-TOP injections; blue), $D$ $=4.69 \mathrm{~nm}$ (8 Te-TOP injections; black), and $D=5.42 \mathrm{~nm}$ (10 Te-TOP injections; red). The absorption maxima were at 660 $\mathrm{nm}$ with 4 injections, $673 \mathrm{~nm}$ with 6 injections, $691 \mathrm{~nm}$ with 8 injections, and $705 \mathrm{~nm}$ with 10 injections.

two-dimensional network of CdTe tetrapods is yielded. We envisage that a three-dimensional network, which is ideal for the electron transport, can be readily achieved via consecutive LB depositions, thereby opening up new ways to utilize them in photovoltaic applications.

\section{RESULTS AND DISCUSSION}

Synthesis and Characterization of CdTe Tetrapods. Using the high temperature organometallic synthesis route, where the solvent, ligands, and cadmium oxide (CdO) are heated with the tellurium (Te) precursor later injected (see Experimental Section), CdTe tetrapods were synthesized. This method involves multiple injections of the Te precursor, up to 10 , effectively separating the nucleation and growth stages. Figure $1 a-d$ shows the TEM images of CdTe tetrapods synthesized via multiple injections of the Te precursor, with 4, 6, 8, and 10 injections, respectively. A close up of a single tetrapod from the 10-injection aliquot is shown in Figure 1e. It should be noted that no size-selection precipitation is conducted and the multiple injection samples are from the same reaction, that is, aliquots were taken directly before the next injection.

As evidenced in the TEM images, samples consist of tetrapods (four arms) and few other-branched structures (two and three arms). In the 10-injection batch (Figure 1d), the diameter of the arms is $5.42 \pm 0.75 \mathrm{~nm}$ with an arm length of $46.7 \pm 9.3 \mathrm{~nm}$. By having multiple injections, one reaction can produce a range of aspect ratios. In the same multiple injection reaction, aliquots were taken after 4, 6, and 8 injections, with the final 10-injection sample allowed to cool in the reaction vessel. Analysis of the 4, 6, 8, and 10-injection samples show that the tetrapods had constant length but increasing arm diameter with increasing injections (Figure 1). The 4, 6, 8, and 10-injection samples had lengths of $40.9 \pm 12,47.2 \pm 5.0,46.6 \pm 7.9$, and $46.7 \pm 9.3$ $\mathrm{nm}$, respectively. Interestingly, the arm width increased starting at $3.85 \pm 1.63 \mathrm{~nm}$ with 4 injections, $3.91 \pm$ $0.52 \mathrm{~nm}$ with 6 injections, $4.69 \pm 0.77 \mathrm{~nm}$ with 8 injections, and $5.42 \pm 0.75 \mathrm{~nm}$ with 10 injections. The relatively wide distribution of the four-injection sample may be due to the initial nucleation of the tetrapods from solution.

Figure 2 shows the absorption spectra from the different injection samples. As evidenced, the absorption peak red-shifted with increasing number of injections, that is, $660 \mathrm{~nm}$ with 4 injections (diameter of arms, $D=$ $3.85 \mathrm{~nm}), 673 \mathrm{~nm}$ with 6 injections $(D=3.91 \mathrm{~nm}), 691$ $\mathrm{nm}$ with 8 injections $(D=4.69 \mathrm{~nm})$, and $705 \mathrm{~nm}$ with 10 injections $(D=5.42 \mathrm{~nm})$. The increase in the number of injections alters the width of the tetrapods, which controlled the absorption maximum. This is consistent with nanorods, where, for example, CdSe rods with a diameter of $3 \mathrm{~nm}$ had an absorption maximum at 650 $\mathrm{nm}$, but increasing the diameter to $7 \mathrm{~nm}$ increased the absorption maximum to $720 \mathrm{~nm} .{ }^{1}$ Clearly, the synthesis 
with multiple injections provides a simple and direct way to control the absorption maximum of the nanocrystals.

The formation of nanocrystals with constant length yet increasing diameter ${ }^{22}$ can be rationalized as follows. During the first injection of Te precursor, nanocrystals are formed in a nearly spherical shape. It is believed that the reaction conditions favored the zinc blende structure in the present study. The zinc blende phase dominates at lower temperatures; with the initial room-temperature injection, the reaction temperature drops considerably, promoting this phase. This thus allows the branched structures to form, as the (111) facet of zinc blende is the atomistic equivalent to the (0001) facet of the wurtzite crystal structure..$^{23}$ It has been reported that multiple twinning faults in the wurtzite crystal structure have led to tetrapods and branched nanocrystals. ${ }^{24}$ This, however, is not believed to be the case in this study, as the twinning was induced by the addition of methylphosphonic acid. With the ligands and precursors used in this study (see Experimental Section), there are no molecules that could assist the twinning phenomenon. ${ }^{24}$

With the second and subsequent injections, the reaction conditions favored the wurtzite crystal structure, which grew from the (111) facet of the zinc blende nuclei forming a stacking fault with the (0001) wurtzite plane. Initially, the $(000 \overline{1})$ facet will grow the quickest, because of the high chemical potential. ${ }^{22,25}$ It is known that nanocrystal growth is diffusion control, 22,25 and the use of longer complex-forming ligands slows the diffusion. As such, the same Cd precursor concentration in a complex with a shorter ligand will diffuse faster than a longer ligand-Cd complex. As a longer ligand (i.e., a 14 carbon chain; tetradecylphosphonic acid, TDPA) was utilized in the study as the Cd complex ligand, the diffusion of the complex (i.e., Cd-TDPA) would be decreased relative to other systems.

It is well-known that at a large monomer concentration, one-dimensional (1D) growth occurs at the higher chemical potential faces (i.e., (0001) in the wurtzite crystal structure). ${ }^{22,23,25,26}$ This is due to the high concentration that combats the slow diffusion of monomer species. At concentrations below this stage, threedimensional (3D) growth takes place where the $\mathrm{Cd}$ complex can diffuse into the vicinity of the growing nanocrystal at the same rate as it is attached to the growing faces. Thus, the crystal maintains its aspect ratio while growing in size. At concentrations below 3D growth, anisotropic growth is halted and ripening occurs in the nanocrystal synthesis. As a result, monomers are removed from the nanocrystals' reactive ends and attach to the short axis, creating more spherical nanoparticles. ${ }^{22,25,27}$ To keep the monomer concentration high, high starting concentrations with relatively short reaction times have been mostly utilized; however, multiple injections have been utilized as well. ${ }^{27,28}$

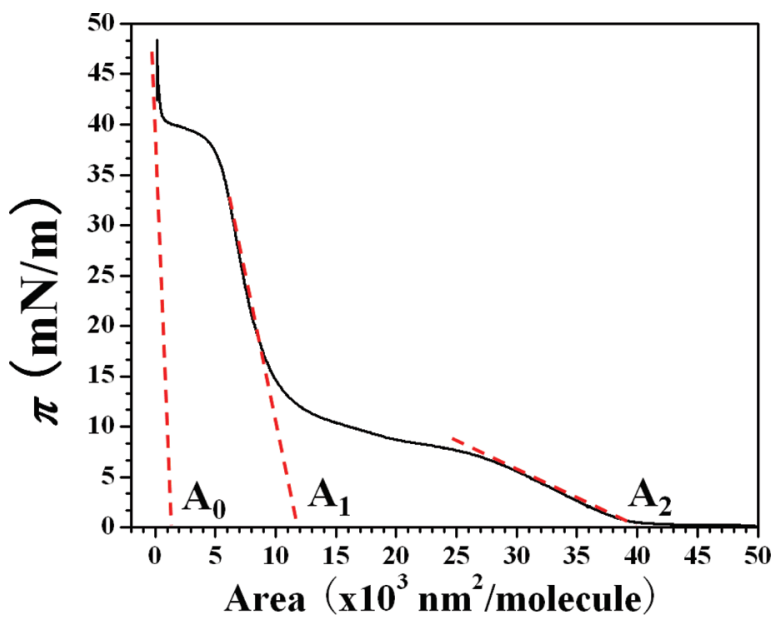

Figure 3. Langmuir isotherm of CdTe tetrapods consisting of the arm length of $46.7 \pm 9.3 \mathrm{~nm}$ and the arm diameter of $5.42 \pm 0.75 \mathrm{~nm}$, that is, the 10-injection sample. Three limiting areas can be identified by extrapolating the pressure rise on the isotherm, $A_{2}=39500 \mathrm{~nm}^{2}, A_{1}=11450 \mathrm{~nm}^{2}$, and $A_{0}=1250 \mathrm{~nm}^{2}$.

In the present study, since statistical analysis of the samples showed no change in length, the monomer concentration was not conducive solely to $1 \mathrm{D}$ or $3 \mathrm{D}$ growth. The possible mechanism is that at the reaction conditions experienced, tetrapods grew to have arm lengths in the $47 \mathrm{~nm}$ range rather quickly, within the first few injections where no aliquots were taken. By the fourth injection, the concentration may have increased enough to allow for growth initially, but by the end of the two minute growth period (right before another injection of precursor), the concentration had decreased and ripening (i.e., the increase in diameter) was occurring. Likewise, subsequent injections promoted growth initially but then gave way to ripening. Close inspection shows that the 4-injection sample has relatively straight arms; however, later samples, especially 8- and 10injections, have a "pearl necklace" appearance, indicative of ripening along the arms. With this, the rate of diffusion of the monomer species was slow enough to provide a delicate balance of reaction conditions, making it possible to increase the diameter of the tetrapod arms while keeping the length constant.

Self-Assembly of CdTe Tetrapods at Air/Water Interface. Langmuir-Blodgett (LB) studies were conducted on the 10-injection sample ( $D=5.42 \mathrm{~nm})$. After purification by centrifuging twice, the tetrapods, at a known volume and concentration (see Experimental Section), were gently placed on the water subphase in the LB trough. The surface pressure was monitored while the surface area was decreased, and the Langmuir isotherm was successfully obtained (Figure 3). Three limiting areas can be determined by extrapolating the increasing pressure slope: $A_{2}=39500 \mathrm{~nm}^{2}, A_{1}=11450 \mathrm{~nm}^{2}$ and $A_{0}=1250 \mathrm{~nm}^{2}$. One tetrapod in isolation should occupy approximately $6166 \mathrm{~nm}^{2}$ given a $44.3 \mathrm{~nm}$ radius (47 $\mathrm{nm}$ arm length in tetrapod). For the limit- 

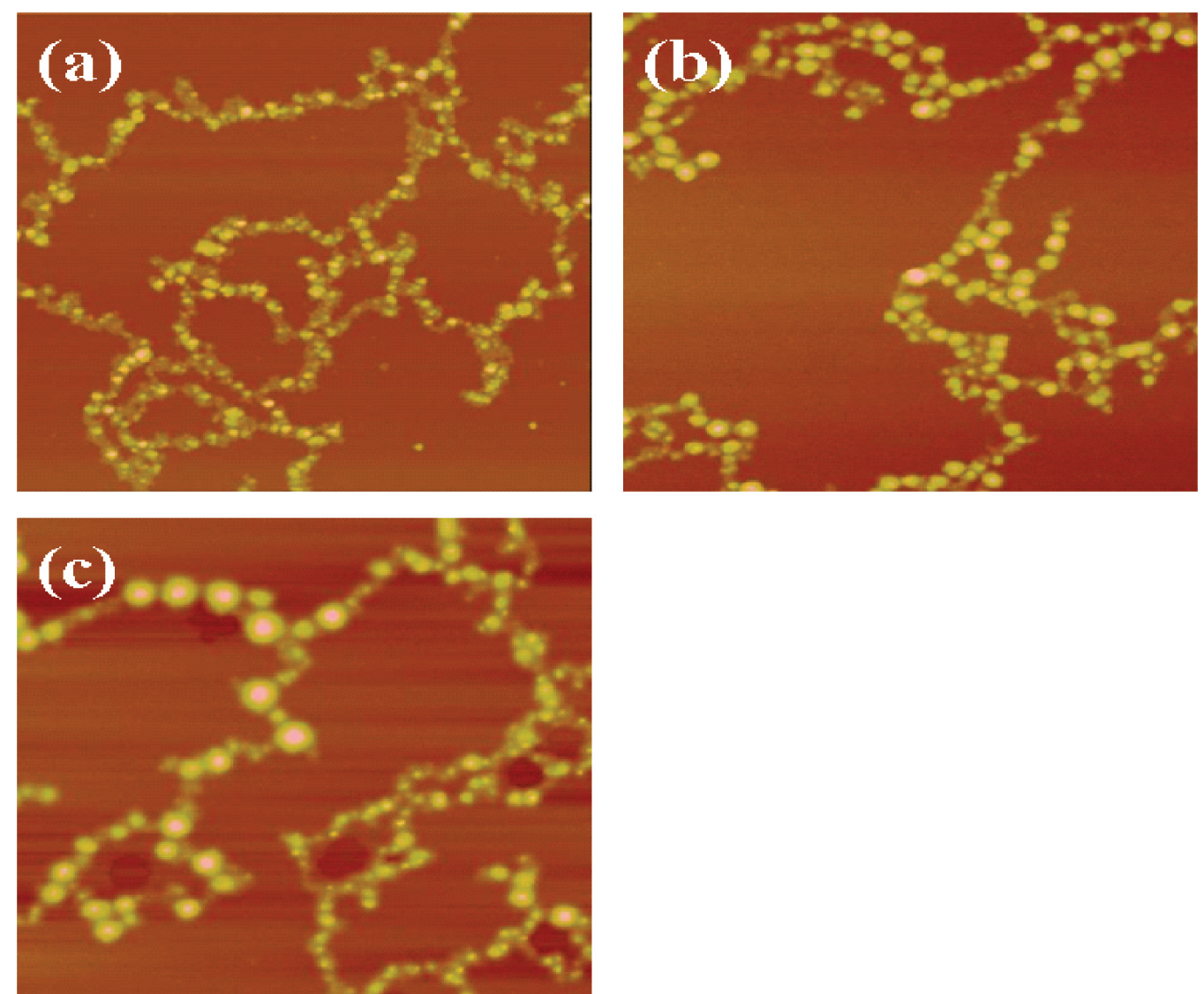

Figure 4. AFM images of CdTe monolayers obtained at surface pressure (a) $\pi=1 \mathrm{mN} / \mathrm{m}$, (b) $\pi=10 \mathrm{mN} / \mathrm{m}$, and (c) $\pi=22$ $\mathrm{mN} / \mathrm{m}$. Scan size $=5 \times 5 \mu \mathrm{m}^{2}$. Z-scale $=100 \mathrm{~nm}$.

ing area $A_{0}$, the tetrapods must interdigitate due to an almost five times reduction in area from the isolated tetrapod $(6166 / 1250=c a .4 .9)$. Clearly, at the onset of pressure (i.e., $A_{2}$ ) and the pressure corresponding to $A_{1}$, the tetrapods are not in a closely packed, spherical arrangement, but rather they coalesce into larger molecules with large voids between

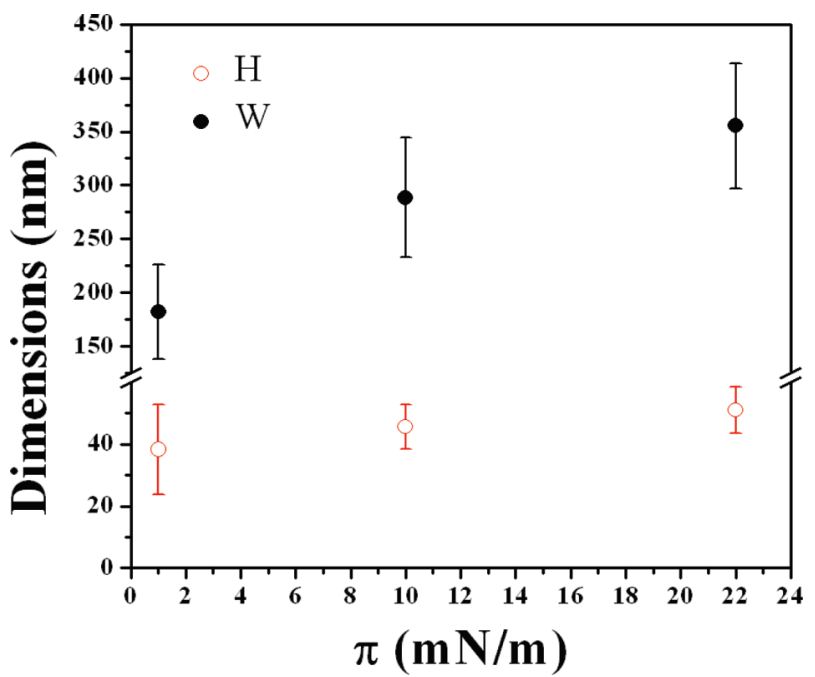

Figure 5. Analysis of height and width of AFM images obtained at different deposition pressures. them. This differs from the well-known gas-liquid-solid phase transition model. ${ }^{29}$

In this context, to scrutinize the exact packing of the tetrapods, LB depositions were conducted at various pressures, specifically $1,10,22$, and $39 \mathrm{mN} \mathrm{m}^{-1}$. Representative atomic force microscopy (AFM) images of the LB depositions are shown in Figure 4 with a scan size of $5 \times 5 \mu \mathrm{m}^{2}$ of the 1,10 , and $22 \mathrm{mN} \mathrm{m}^{-1}$ samples. No AFM images were obtained on the $39 \mathrm{mN} \mathrm{m}^{-1}$ sample due to the extreme roughness of the sample. It is evident that ribbon-like morphologies of tetrapods occur at the onset of pressure, that is, $1 \mathrm{mN} \mathrm{m}^{-1}$. This morphology would create the increase in pressure at a larger packing area due to the presence of voids in the monolayer. Additionally, aggregates can be seen along the ribbon, representing closer packing and possible nucleation sites (Figure 4). The formation of ribbons is attributed to the dewetting of the CdTe nanocrystals and the unique tetrapod shape. The dewetting mechanism is widely proposed to account for the selfassembly at the air/water interface, ranging from polymers ${ }^{30}$ to nanocrystals. ${ }^{31}$ The dewetting arises from the hydrophobic TDPA ligands attached to the nanocrystals. Upon solvent evaporation, these ligands were intimately in contact with the water subphase. Since no amphiphilic molecules were present to stabilize the sys- 

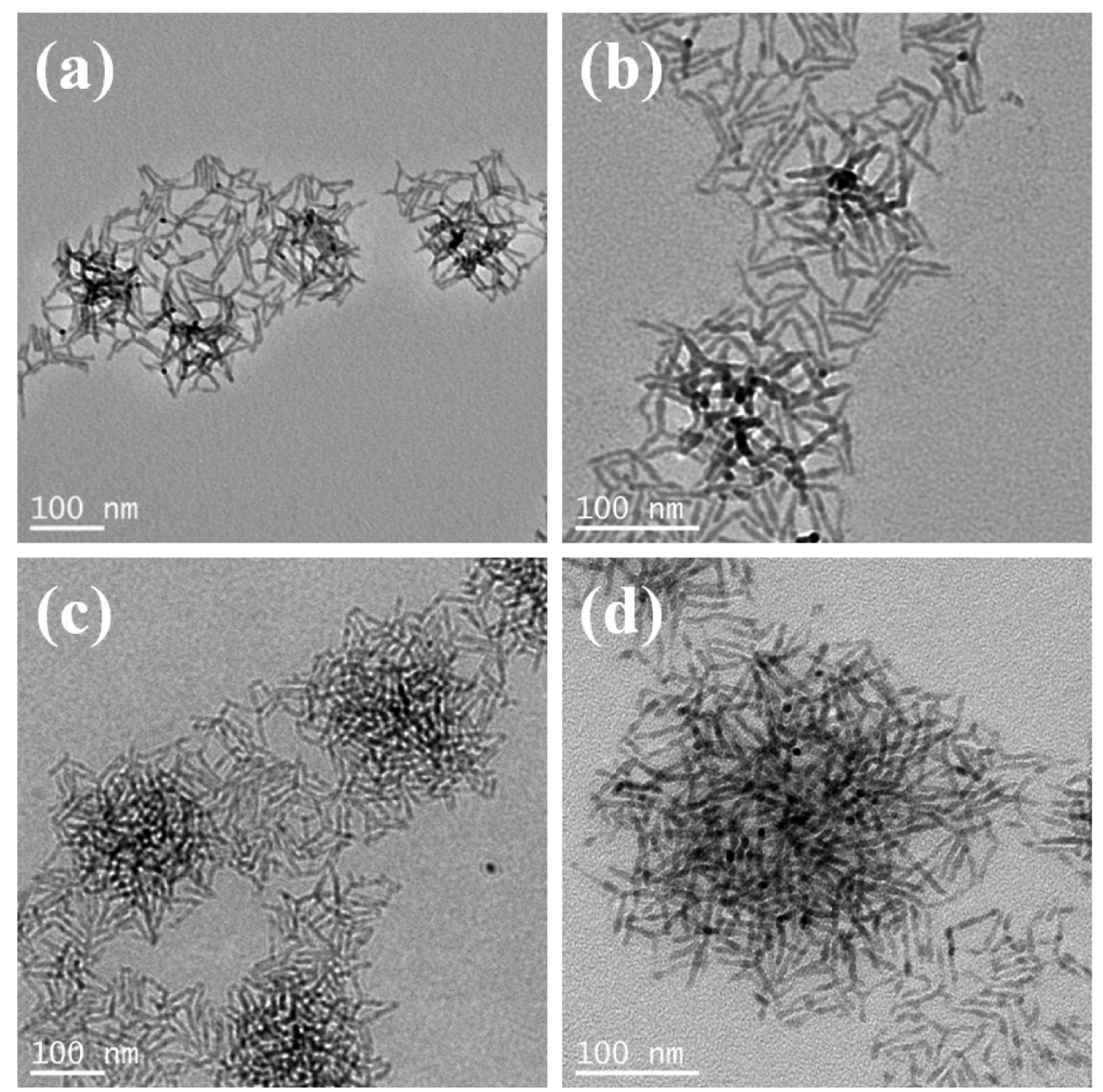

Figure 6. TEM images of CdTe tetrapods obtained at the different deposition pressures. (a) $\pi=1 \mathrm{mN} / \mathrm{m}$, (b) $\pi=10 \mathrm{mN} / \mathrm{m}$, (c) $\pi=22 \mathrm{mN} / \mathrm{m}$, and (d) $\pi=39 \mathrm{mN} / \mathrm{m}$.

tem, the liquid thin film of TDPA-capped nanocrystals at the water surface becomes unstable, leading to the dewetting (i.e., rupturing) of the liquid film to generate randomly distributed holes. The holes then grow and the rims ahead of the holes merged to form aggregates. However, because of the unique tetrapod shape, the close packing of the nanocrystals could not occur. Instead, arms of two tetrapods could closely pack, with branching possible along other arm directions. Since the nanocrystals are rigid, rearrangement of the tetrapods to form long fibers, as in flexible polymers, ${ }^{32}$ cannot happen. Consequently, the ribbon and aggregate structures emerge, thereby minimizing the system energy.

Comparing the AFM images in Figure 4, it is not possible to discern any significant difference between the 1 and $10 \mathrm{mN} \mathrm{m}^{-1}$ samples. In light of this, analysis was done on the AFM images, measuring the height and width of the ribbons across the aggregates (Figure 5). It is clear that the height does not increase significantly in the $10 \mathrm{mN} \mathrm{m}^{-1}$ deposition. This is expected to be the case, as the ribbons should be a monolayer of the nanocrystals. In fact, the fourth tetrapod arm, which has the possibility to be normal to the substrate, would have a height of approximately $47 \mathrm{~nm}$, falling within one standard deviation of the measurements (Figure 5). Similar to the 1 and $10 \mathrm{mN} \mathrm{m}^{-1}$ depositions, the $22 \mathrm{mN} \mathrm{m}^{-1}$ deposition had a height that only slightly increased. In all three pressures, the width of the aggregates increased from the low to high pressure (Figures 4 and 5).

It is noteworthy that large aggregates along the ribbon are clearly visible in the $22 \mathrm{mN} \mathrm{m}^{-1}$ sample (Figure $4 \mathrm{c}$ ). The $22 \mathrm{mN} \mathrm{m}^{-1}$ deposition is in the middle of the sharp rise in pressure, resulting in the limiting area $A_{1}$ of $11450 \mathrm{~nm}^{2}$. During this sharp rise, the increased number of aggregates could be responsible for the increase in pressure, as the tetrapods can no longer easily pack into the aggregates. These larger morphologies are dispersed throughout the ribbon structure and responsible for the larger width seen in the AFM analysis (Figure 5). The deposition at $22 \mathrm{mN} \mathrm{m}^{-1}$ shows the larger aggregate morphology similar to the deposition at $10 \mathrm{mN} \mathrm{m}^{-1}$; however, the aggregates have condensed and thickened, resulting in a denser networklike pattern.

Comparing the analyzed ribbon widths shows a clear trend among the samples. At the higher pres- 

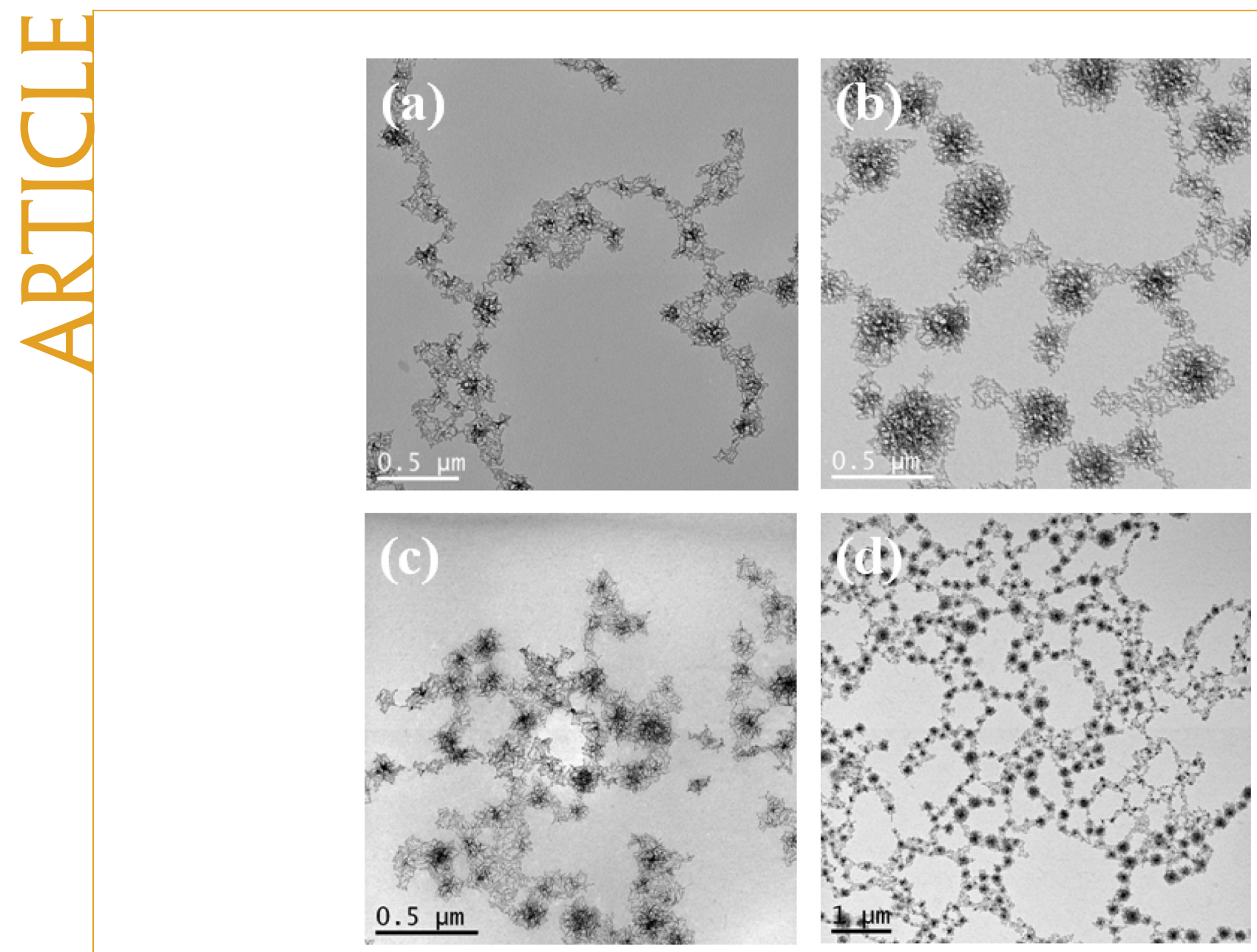

Figure 7. Large scale TEM images obtained at pressures of (a) $\pi=1 \mathrm{mN} / \mathrm{m}$, (b) $\pi=10 \mathrm{mN} / \mathrm{m}$, (c) $\pi=22 \mathrm{mN} / \mathrm{m}$, and (d) $\pi$ $=39 \mathrm{mN} / \mathrm{m}$.

sure, more nanocrystals coalesce into the ribbons, either through simple migration of particles or two ribbons combining. The packing of the nanocrystals is the densest in the aggregates, possibly indicating the sites of ribbon nucleation. In these aggregates, nanocrystals could overlap each other due to the branching of the tetrapods. Due to the tetrapod shape, the three arms of the tetrapod would not lie on a flat plane with $120^{\circ}$ between them but rather be protruding at $109.5^{\circ}$. This would allow other nanocrystal arms to slide underneath the central, raised portion of a tetrapod, resulting in a stacked structure. However, as seen in the AFM height analysis, this stacking does not drastically increase the height of the ribbons.

During AFM imaging, it was found that the monolayer could be seen visually with optical microscopy (OM; images shown in Supporting Information, Figure S1). In the optical micrographs, larger voids on a length scale of a hundred micrometers in diameter can be seen, neighboring more concentrated nanocrystal regions. This is further evidence of the dewetting phenomenon; moreover, AFM section analysis revealed bare silicon substrate in the voids (data not shown). This is in contrast to our previous work performed on CdTe nanorods, where excess capping ligands were responsible for the increased surface area. $^{20}$

To examine individual nanocrystals in the monolayer packing, transmission electron microscopy (TEM) imaging was conducted on LB samples produced on TEM grids as shown in Figure 6 (close-up) and Figure 7 (large-scale). In Figure 6, the packing of individual tetrapods can be seen, with a trend of tighter packing with increasing pressure. Furthermore, small aggregates along the ribbon can be seen, which start as small clusters of few tetrapods at $1 \mathrm{mN} \mathrm{m}^{-1}$ and grow to clusters of hundreds at the highest pressure, $39 \mathrm{mN} \mathrm{m}^{-1}$. As $10 \mathrm{mN} \mathrm{m}^{-1}$ was in the middle a pressure plateau, there is reorganization occurring with the tetrapods going from ribbon morphologies (Figure 7a) to larger cluster morphologies (Figure 7b). These larger cluster morphologies have tetrapods more closely packed than the ribbon structures, with more pushed onto other tetrapods (Figure $7 \mathrm{~b}$ ). This trend continues through higher pressures, 22 and $39 \mathrm{mN} \mathrm{m}^{-1}$. At the highest pressure (39 $\mathrm{mN} \mathrm{m}^{-1}$ ), the network structure was formed successfully to minimize the energy and the surface area by interdigitation of the tetrapod arms. As the tetrapods form the larger cluster and network structures, the tetrapods interdigitate with extreme packing. 
Close inspection of the $39 \mathrm{mN} \mathrm{m}^{-1}$ deposition revealed that it exhibits a clear picture of the aggregated cluster, composed of a majority of tetrapods, surrounded by less branched nanocrystals (Figure $6 \mathrm{~d}$ ). This is probably due to the ease of closer packing in the less branched structures compared to the fully branched tetrapods. On the other hand, compared to the samples at lower pressures $\left(1,10\right.$, and $\left.22 \mathrm{mN} \mathrm{m}^{-1}\right)$, the extreme packing in the $39 \mathrm{mN} \mathrm{m}^{-1}$ sample is due most likely to the combination of high surface pressure and the tetrapod shape. The tetrapods with the fourth arm along the electron beam axis are easily seen due to the darker contrast. The increase the pressure beyond $39 \mathrm{mN} \mathrm{m}^{-1}$ results in a quick, sharp rise in pressure followed by the collapse of the monolayer. This indicates the network structure at $39 \mathrm{mN} \mathrm{m}^{-1}$ is the final stable morphology. Since the monolayer at $39 \mathrm{mN} \mathrm{m}^{-1}$ is stable, the overlapping of nanocrystals is primarily due to the tetrapod shape, where the nanocrystals cannot lie flat on the water subphase. This leaves voids beneath the nanocrystals, allowing other nanocrystals to penetrate. At high enough pressure, that is, $39 \mathrm{mN} \mathrm{m}^{-1}$, the penetrating nanocrystals can start to slightly push up the tetrapods while still keeping the monolayer network intact. Notably, for the $39 \mathrm{mN} \mathrm{m}^{-1}$ sample, the ribbon morphologies condensed, forming a thicker network structure. There exists a hierarchal arrangement over three length scales in the network structure (Supporting Information, Figure S1d). On the submicrometer scale, tetrapods form a network structure with voids with a magnitude of one micrometer (Figure $7 d$ and Supporting Information, Figure S2). This network morphology grows into a larger network with voids on the order of tens of micrometers in diameter (Figure $7 d$ and Supporting Information, Figure S1d), and again to a net- work with voids on the hundred micrometers range, as shown in the optical micrograph (Supporting Information, Figure S1d).

\section{CONCLUSIONS}

CdTe tetrapods were successfully synthesized using a modified procedure. This procedure utilizes multiple injections of the Te precursor, resulting in increased arm diameter instead of increased length. By increasing the arm diameter, the absorption maximum red-shifted, which is desirable for optoelectronic applications, such as photovoltaics. This process provides a simple synthesis with multiple and tunable products by taking aliquots at different time and injection intervals. Subsequently, the tetrapods with an arm diameter of $5.42 \mathrm{~nm}$ and length of $46.7 \mathrm{~nm}$ were placed at the air/water interface, exhibiting a dewetting behavior. The dewetting and ribbon formation are attributed to the hydrophobic nature of ligand-functionalized nanocrystals and their unique tetrapod shape. Langmuir-Blodgett depositions were conducted at different surface pressures with their packing behaviors scrutinized by a combination of AFM, TEM and optical microscopy studies. The tetrapods form a ribbon structure at the onset of pressure, presumably due to the tetrapod shape. At highest surface pressure, these ribbons coalesce into a network structure, yielding a hierarchical structure that has voids on the submicrometer, tens, and hundreds of micrometer scale. These intriguing network structures can serve as a building block, by improving optical absorption and electron transport, for potential applications in hybrid solar cells due to their unique, continuous pathway, controllable absorption maximum, and semiconducting properties.

\section{EXPERIMENTAL SECTION}

Synthesis of CdTe Tetrapods. Chemicals were purchased from various sources, including Fisher Scientific, PCI Synthesis, SigmaAldrich, Strem Chemicals, and TCl America, and used as received. $\mathrm{CdTe}$ tetrapods were synthesized by modifying a method for $\mathrm{CdTe}$ nanorods in the literature. ${ }^{28}$ First, $0.114 \mathrm{~g}$ of cadmium oxide (CdO), $0.43 \mathrm{~g}$ of tetradecylphosphonic acid (TDPA), and $7 \mathrm{~g}$ of trioctylphosphine oxide (TOPO) were degassed in a three-neck flask for $1 \mathrm{~h}$ at $120^{\circ} \mathrm{C}$ before increasing the temperature to above $300^{\circ} \mathrm{C}$, where the $\mathrm{CdO}$ completely reacted and the solution turned clear. The temperature was decreased to $290{ }^{\circ} \mathrm{C}$, and 0.5 $\mathrm{mL}$ of tellurium -trioctylphosphine (Te-TOP) solution, $c=0.2$ $M$, was injected. After the initial injection, the temperature was increased to $300^{\circ} \mathrm{C}$, and subsequent $0.5 \mathrm{~mL} \mathrm{Te}-\mathrm{TOP}$ solutions were injected every 2 min with the width of arms of CdTe tetrapods controlled by the number of injections. In this work, the total number of injections was 10 , and after the injections heat was removed. The nanocrystals were precipitated twice with ethanol and dissolved in hexane or chloroform.

Langmuir Isotherms and LB Depositions. Highly polished Si wafers (Okmetic) with an orientation of [111] were immersed in sulfuric acid with NoChromix oxidizer (Godax Laboratories) for a minimum of $6 \mathrm{~h}$. Afterward, the wafers were rinsed a minimum of three times with NanoPure water, blown dry with $\mathrm{N}_{2}$, and used as LB substrates. Langmuir isotherms and LB depositions were

www.acsnano.org conducted on an R \& K-1 trough (Riegel \& Kirstein, $\mathrm{GmbH}$ ). In a typical experiment, the tetrapod chloroform solution, $c=0.61$ $\mathrm{mg} / \mathrm{mL}\left(3.2 \times 10^{-8} \mathrm{M}\right)$, was placed on the water surface (NanoPure, $\rho>18 \mathrm{M} \Omega \mathrm{cm}$ ), totaling $30-60 \mu \mathrm{L}$. After complete solvent evaporation, typically $30-45 \mathrm{~min}$, Langmuir isotherms were recorded while the surface was compressed at a rate of 150 $\mu \mathrm{m} / \mathrm{s}$. For LB depositions, the pressure was held constant while lifting the clean $\mathrm{Si}$ substrate/TEM grid from the water subphase at a rate of $35 \mu \mathrm{m} / \mathrm{s}$.

Characterizations. TEM imaging was conducted on a JEOL 2100 scanning transmission electron microscope (STEM) operated at 200 kV at MNIF of lowa State University. In a typical setup, a solution was drop cast onto a TEM grid (carbon-coated 400-mesh copper used as received (Electron Microscopy Sciences)), after which, the solvent was allowed to evaporate at room temperature. Atomic force microscopy (AFM) images were taken in tapping mode on a Dimension 3000 (Veeco) instrument. BS-tap300 tips (Budget Sensors) with spring constants ranging from 20 to $75 \mathrm{~N} / \mathrm{m}$ were used as scanning probes. Scan rates were kept between 0.6 and $1.1 \mathrm{~Hz}$ for all imaging. Optical microscopy was taken on an Olympus BX51 instrument in reflection mode on the LB monolayers deposited on Si wafers.

Acknowledgment. We gratefully acknowledge support from the National Science Foundation (Grant NSF-CBET 0824361). 
Supporting Information Available: Optical micrographs of LB samples obtained at different surface pressures and TEM images of LB sample at the surface pressure of $39 \mathrm{mN} / \mathrm{m}$. This material is available free of charge via the Internet at http://pubs.acs.org.

\section{REFERENCES AND NOTES}

1. Huynh, W. U.; Dittmer, J. J.; Alivisatos, A. P. Hybrid Nanorod-Polymer Solar Cells. Science 2002, 295, 2425-2427.

2. Gur, I.; Fromer, N. A.; Geier, M. L.; Alivisatos, A. P. Air-stable All-Inorganic Nanocrystal Solar Cells Processed from Solution. Science 2005, 310, 462-465.

3. Kang, Y. M.; Park, N. G.; Kim, D. W. Hybrid Solar Cells with Vertically Aligned CdTe Nanorods and a Conjugated Polymer. Appl. Phys. Lett. 2005, 86, 113101.

4. Xu, J.; Wang, J.; Mitchell, M.; Mukherjee, P.; Jeffries-EL, M.; Petrich, J. W.; Lin, Z. Q. Organic-Inorganic Nanocomposites via Directly Grafting Conjugated Polymers onto Quantum Dots. J. Am. Chem. Soc. 2007, 129, 12828-12833.

5. Xu, J.; Xia, J.; Lin, Z. Q. Evaporation-Induced Self-Assembly of Nanoparticles from a Sphere-on-Flat Geometry. Angew. Chem., Int. Ed. 2007, 46, 1860-1863.

6. Lin, Z. Q. Organic-Inorganic Nanohybrids via Direct Tailoring Semiconductor Nanocrystals with Conjugated Polymers. Chem. - Eur. J. 2008, 14, 6294-6301.

7. Goodman, M. D.; Xu, J.; Wang, J.; Lin, Z. Q. Semiconductor Conjugated Polymer-Quantum Dot Nanocomposites at the Air/Water Interface and Their Photovoltaic Performance. Chem. Mater. 2009, 21, 934-938.

8. Xu, J.; Xia, J. F.; Wang, J.; Shinar, J.; Lin, Z. Q. Quantum Dots Confined in Nanoporous Alumina Membranes. Appl. Phys. Lett. 2006, 89, 133110.

9. Wang, J.; Xu, J.; Goodman, M. D.; Chen, Y.; Cai, M.; Shinar, J.; Lin, Z. Q. A Simple Biphasic Route to Water Soluble Dithiocarbamate Functionalized Quantum Dots. J. Mater. Chem. 2008, 18, 3270-3274.

10. Colvin, V. L.; Schlamp, M. C.; Alivisatos, A. P. Light-Emitting Diodes Made from Cadmium Selenide Nanocrystals and a Semiconducting Polymer. Nature 1994, 370, 354-357.

11. Klimov, V. I.; Mikhailovsky, A. A.; Xu, S.; Malko, A.; Hollingsworth, J. A.; Leatherdale, C. A.; Eisler, H. J.; Bawendi, M. G. Optical Gain and Stimulated Emission in Nanocrystal Quantum Dots. Science 2000, 290, 314-317.

12. Kimura, J.; Maenosono, S.; Yamaguchi, Y. Near-Field Optical Recording on a CdSe Nanocrystal Thin Film. Nanotechnology 2003, 14, 69-72.

13. Lee, J.; Govorov, A. O.; Kotov, N. A. Bioconjugated Superstructures of $\mathrm{CdTe}$ Nanowires and Nanoparticles: Multistep Cascade Forster Resonance Energy Transfer and Energy Channeling. Nano Lett. 2005, 5, 2063-2069.

14. Chan, W. C. W.; Nie, S. Quantum Dot Bioconjugates for Ultrasensitive Nonisotopic Detection. Science 1998, 281, 2016-2018.

15. Medintz, I. L.; Uyeda, H. T.; Goldman, E. R.; Mattoussi, H. Quantum Dot Bioconjugates for Imaging, Labelling and Sensing. Nat. Mater. 2005, 4, 435-446.

16. Alivisatos, A. P. The Use of Nanocrystals in Biological Detection. Nat. Biotechnol. 2004, 22, 47-52.

17. Fu, A.; Gu, W.; Boussert, B.; Koski, K.; Gerion, D.; Manna, L.; LeGros, M.; Larabell, C. A.; Alivisatos, A. P. Semiconductor Quantum Rods As Single Molecule Fluorescent Biological Labels. Nano Lett. 2007, 7, 179-182.

18. Hu, J.; Li, L.; Yang, W.; Manna, L.; Wang, L.; Alivisatos, A. P. Linearly Polarized Emission from Colloidal Semiconductor Quantum Rods. Science 2001, 292, 2060-2063.

19. Kim, F.; Kwan, S.; Akana, J.; Yang, P. D. Langmuir-Blodgett Nanorod Assembly. J. Am. Chem. Soc. 2001, 123, 4360-4361.

20. Zimnitsky, D.; Xu, J.; Lin, Z. Q.; Tsukruk, V. V. Domain and Network Aggregation of CdTe Quantum Rods within Langmuir-Blodgett Monolayers. Nanotechnology 2008, 19, 215606.

21. He, J.; Zhang, Q.; Gupta, S.; Emrick, T.; Russell, T. R.;
Thiyagarajan, P. Drying Droplets: A Window into the Behavior of Nanorods at Interfaces. Small 2007, 3, 12141217.

22. Shieh, F.; Saunders, A. E.; Korgel, B. A. General Shape Control of Colloidal CdS, CdSe, CdTe Quantum Rods and Quantum Rod Heterostructures. J. Phys. Chem. B 2005, 109, 8538-8542.

23. Peng, Z. A.; Peng, X. G. Mechanisms of the Shape Evolution of CdSe Nanocrystals. J. Am. Chem. Soc. 2001, 123, 1389-1395.

24. Manna, L.; Milliron, D. J.; Meisel, A.; Scher, E. C.; Alivisatos, A. P. Controlled Growth of Tetrapod-Branched Inorganic Nanocrystals. Nat. Mater. 2003, 2, 382-385.

25. Carbone, L.; Kudera, S.; Carlino, E.; Parak, W. J.; Giannini, C.; Cingolani, R.; Manna, L. Multiple Wurtzite Twinning in CdTe Nanocrystals Induced by Methylphosphonic Acid. J. Am. Chem. Soc. 2006, 128, 748-755.

26. Peng, X. G. Mechanisms for the Shape-Control and ShapeEvolution of Colloidal Semiconductor Nanocrystals. Adv. Mater. 2003, 15, 459-463.

27. Manna, L.; Scher, E.; Alivisatos, A. P. Synthesis of Soluble and Processable Rod-, Arrow-, Teardrop-, and TetrapodShaped CdSe Nanocrystals. J. Am. Chem. Soc. 2000, 122, 12700-12706.

28. Peng, X.; Manna, L.; Yang, W. D.; Wickham, J.; Scher, E.; Kadavanich, C.; Alivisatos, A. P. Shape Control of CdSe Nanocrystals. Nature 2000, 404, 59-61.

29. Petty, M. C., Langmuir-Blodgett Films: An Introduction; Cambridge University Press: Cambridge, NY, 1996.

30. Zhao, L.; Goodman, M. D.; Bowden, N. B.; Lin, Z. Q. SelfAssembly of an Ultra-High-Molecular-Weight Comb Block Copolymer at the Air-Water Interface. Soft Matter 2009, 5 , 4698-4703.

31. Muller-Buschbaum, P. Dewetting and Pattern Formation in Thin Polymer Films As Investigated in Real and Reciprocal Space. J. Phys., Condens. Matter 2003, 15, R1549-R1582.

32. Ornatska, M.; Bergman, K. N.; Goodman, M.; Peleshanko, S.; Shevchenko, V. V.; Tsukruk, V. V. Role of Functionalized Terminal Groups in Formation of Nanofibrillar Morphology of Hyperbranched Polyesters. Polymer 2006, 47, 8137-8146. 